\title{
REMOTE AMBIENT VIBRATION MEASUREMENTS WITH REAL- APERTURE RADAR TO ESTIMATE BUILDINGS DYNAMIC PROPERTIES
}

\author{
Rodrigo E. Alva ${ }^{1 *}$, José R. González-Drigo ${ }^{1}$, Guido Luzi², Oriol Caselles ${ }^{1}$, Luís G. Pu- \\ jades $^{1}$, Yeudy F. Vargas-Alzate ${ }^{1}$, Luis A. Pinzón ${ }^{1}$ \\ ${ }^{1}$ Universitat Politècnica de Catalunya, Dep. of Civil and Environmental Engineering \\ C. Jordi Girona 1-3, Campus Nord UPC, Barcelona, Spain. \\ \{rodrigo.esteban.alva, lluis.pujades, jose.ramon.gonzalez, luis.pinzon, yeudy.felipe.vargas\}@upc.edu \\ ${ }^{2}$ Centre Tecnològic de Telecomunicacions de Catalunya (CTTC) \\ Parc Mediterrani de la Tecnologia (PMT) Av. Carl Friedrich Gauss 7, 08860, Castelldefels, Spain \\ guido.luzi@cttc.cat
}

\begin{abstract}
The use of sensors to obtain the dynamic properties of structures by means of ambient vibrations has become a common practice. However, conventional and more extensively used methods require the installation of sensors, which should be in contact with the structure. These methods could be even risky in case of structures weakened by a hazardous event, for which the risk of collapse is unknown. In this study, two main subjects are analyzed and discussed. The former is related to the use of the Real-Aperture Radar (RAR) as a non-invasive, remote sensing interferometric method to measure the vibration response to ambient noise of buildings. As a test bed, two high-rise regular buildings with reinforced concrete frames (8-story) and steel frames (12-story), located in Barcelona, Spain, are analyzed. Notice that the RAR device is a sensor using the principle of interferometry to measure displacements. This type of sensors has exhibited a good performance in urban environments at different atmospheric, meteorological and lighting conditions, compared to other types of remote sensors (e.g. Laser Doppler). Another advantage is that RAR devices have a very good resolution and they can operate at great distances from the object to be measured. However, so far, RAR is rarely used in buildings, being still a poorly studied technique. The latter subject is related to the optimal post-processing of the acquired data. At this respect, the Power Spectral Density (PSD) signal-processing technique is used to process the signals acquired. From this analysis, the predominant periods of the analyzed buildings were obtained and then they were compared with those periods obtained from measurements of accelerometers located inside the structures. The results indicate that the use of RAR can be a reliable alternative to estimate the structural periods in a relatively simple and non-invasive manner.
\end{abstract}

Keywords: Radar, Remote Sensing, Monitoring, Ambient Vibrations, Frequency. 
Rodrigo E. Alva, José R. González-Drigo, Guido Luzi, Oriol Caselles, Luís G. Pujades, Yeudy F. Vargas-Alzate, Luis A. Pinzón

\section{INTRODUCTION}

The sensors most commonly used nowadays to measure the dynamic properties of buildings are the accelerometers. Over the years, these sensors have proven to be accurate enough for assessing the dynamic response of structures to strong and weak motions through continuous monitoring [1]. However, permanent monitoring requires costly installations and continuous maintenance, so this type of monitoring is carried out in a few cases, generally, in new buildings of high importance. Moreover, devices that must be in contact with the structure can be problematic when entering to this one is restricted due to difficulty of access and/or prevented when the structure has symptoms of damage and the risk of collapse is unknown. Thus, the use of non-invasive or remote sensing methods emerges as a reliable and safety alternative, especially in, but not limited to, post-earthquake scenarios. At this respect, the Real Aperture Radar (RAR) is a good candidate to be a device providing a solution to the problem. RAR is a sensor using the principle of interferometry to measure displacements [2]. This type of sensors has exhibited a good performance in urban environments under different atmospheric, meteorological and lighting conditions. Noticeably, these devices perform better than other types of sensors (e.g. Laser Doppler) [3]. Moreover, RAR sensors show a very good resolution even at long distances from the object to be measured. Nevertheless, RAR applications in buildings are poorly studied and rarely used.

In this paper, the results of two case studies, using RAR as monitoring technique, for obtaining the dynamic behavior of buildings are shown. The results obtained with RAR are validated by considering measurements recorded with accelerometers. The main advantages and disadvantages of the RAR-based method as well as the main issues related to the post-processing data are summarized and discussed in detail.

\section{MONITORING TECHNIQUES}

\subsection{Accelerometer}

Over the past years, accelerometer devices have become the standard for monitoring structural vibrations [1]. This fact has motivated an important technological development on these devices making them smaller, simpler and more precise. However, structural monitoring by using accelerometers requires that these sensors be installed inside of, and in contact with, the building. This fact, for safety and ease reasons, restrict the use of accelerometers to healthy and accessible structures. Other limitations can come from the wiring, which is often needed to link sensors and recording units. Additional electronic noise and other difficulties related to the deployment and maintenance of a number of long cables can arise. Nonetheless, these devices provide reliable measurements and, therefore, they will be employed in order to validate the results obtained via the RAR technique. To do so, a high sensitivity tri-axial accelerometer (three orthogonal components) was located at the roof of the buildings studied herein. Samples of about one hour of recording with a sampling rate of $100 \mathrm{~Hz}$ are recorded and processed for each structure.

\subsection{Real-Aperture Radar}

The RAR is a device that was originally implemented to detect ground displacements sampling remotely with radar-equipped satellites [2]. However, in the year 2000 there was a paradigm shift and the RAR was used to measure civil structures [4]. Since then, RAR technology has become popular to monitor infrastructures of different types, especially in large infrastruc- 
Rodrigo E. Alva, José R. González-Drigo, Guido Luzi, Oriol Caselles, Luís G. Pujades, Yeudy F. Vargas-Alzate, Luis A. Pinzón

ture as bridges and towers [5]. For instance, the device used in this study was originally designed to monitor large infrastructures (e.g. bridges, towers, dams and wind turbines towers) by the Italian company IDS (Ingegneria dei Sistemi SpA).

The radar is easily transportable and is mounted on a robust tripod that can be adjusted in height and inclination to aim at the target structure easily. This system consists of a sensor module connected to a portable computer through Universal Serial Bus (USB) connection and the provided software can be used to adjust the sampling parameters. A rechargeable external battery allows up to 5 hours of continuous use. With a precision of $10 \mu \mathrm{m}$, the range of operation can reach up to $1 \mathrm{~km}$ at the minimum sampling frequency of $40 \mathrm{~Hz}$ (the range of operation decreases as the sampling frequency increases up to $200 \mathrm{~Hz}$ ); details on the operation of this device can be found in [6]. An image of the RAR device under recording conditions can be seen in Figure 1.
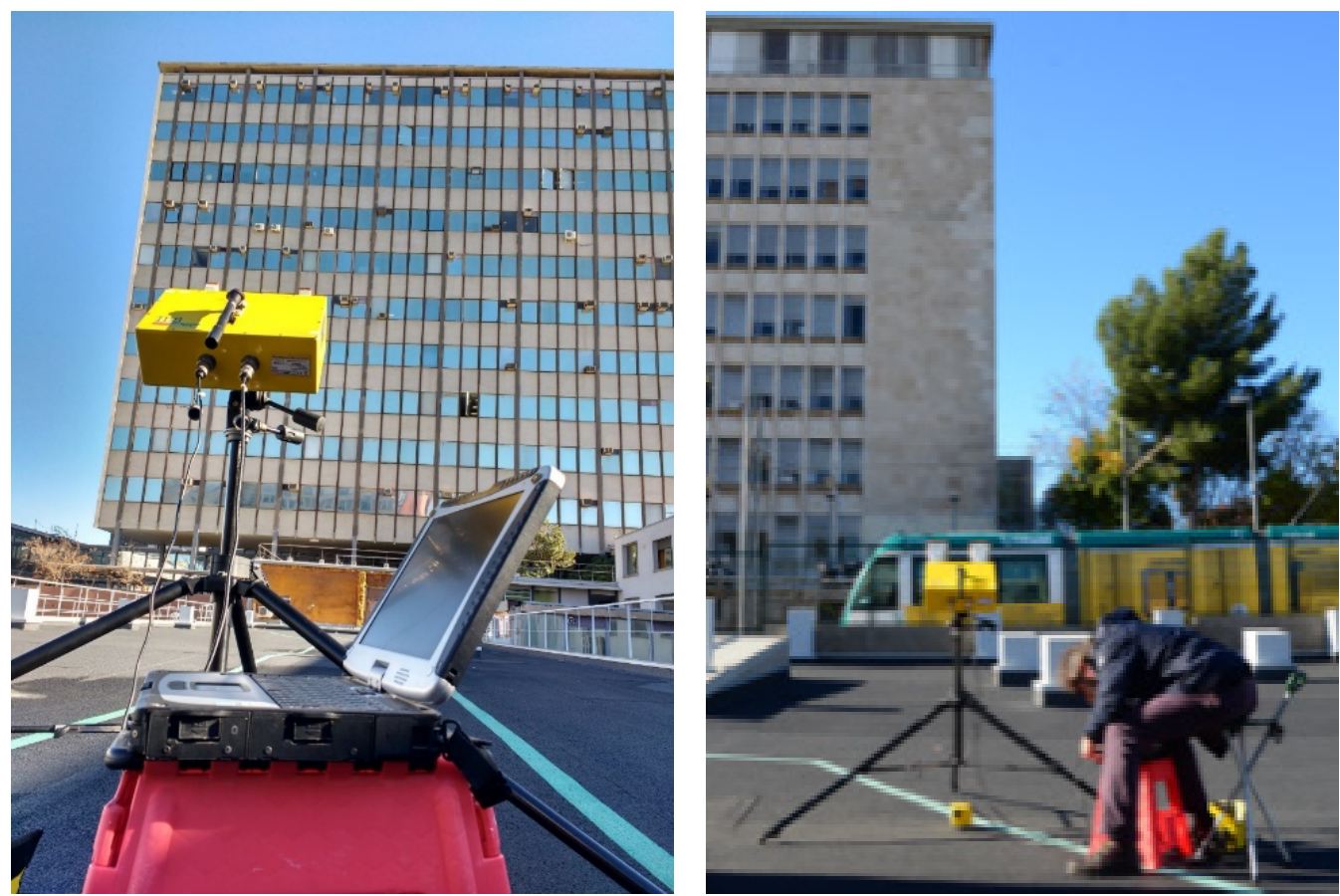

Figure 1: Remote sensing with RAR, building A (left) and building B (right).

A limitation of RAR devices is that they can measure only displacements in the direction of its line of sight (LOS). This fact implies that only one direction of the structure can be monitored at a time. Thus, assuming that the vertical displacement is not significant, the recorded signal corresponds mainly to the LOS component of the displacement of the building in the horizontal direction.

\section{CASE STUDIES}

The first case of study, building B in Figure 1, is a reinforced concrete 8-story building with a rectangular plan of around $58 \times 19 \mathrm{~m}$. The second case of study, building A in Figure 1, is a 12-storey steel structure with a U-shaped floor plan consisting of two well-differentiated structural systems: the central and lateral ones. The two lateral structures are symmetrical with each other and have a lengthened plant of $59 \times 13 \mathrm{~m}$ that is not rectangular, but thinner towards both ends. These three structures are detached from each other and this investigation is focused only in the lateral body shown in Figure 1 (see also Figure 2). 
Rodrigo E. Alva, José R. González-Drigo, Guido Luzi, Oriol Caselles, Luís G. Pujades, Yeudy F. Vargas-Alzate, Luis A. Pinzón

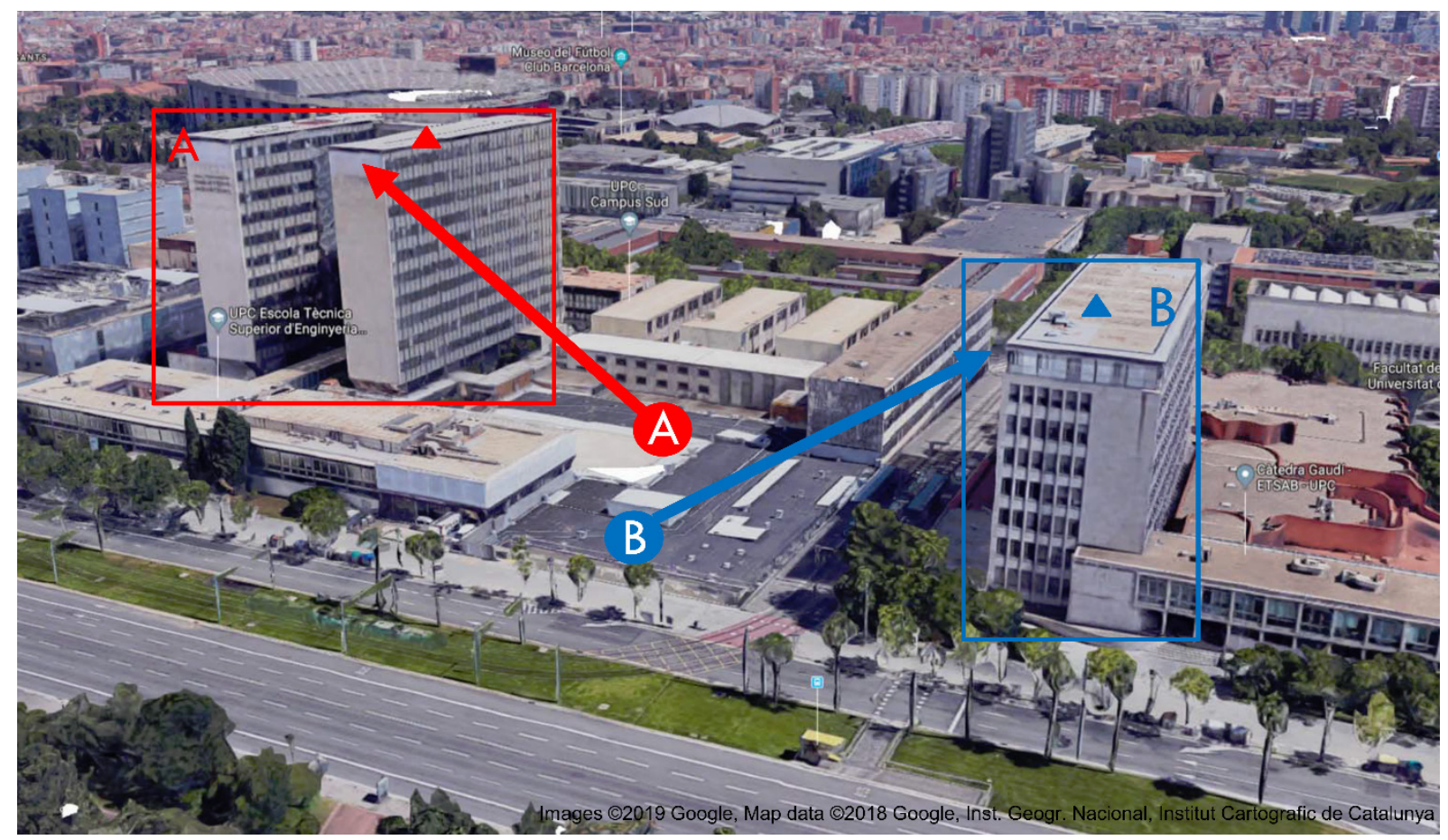

Figure 2: Building A (left) and building B (right), the circles indicate the sites where the RAR device was located and arrows correspond to the LOS. Triangles show where the accelerometers were placed.

As pointed out above, acceleration measurements were made at the roofs of the buildings by means of a tri-axial accelerometer placed near the center of rigidity. The accelerometers recorded the vibration of the buildings due to ambient noise in the three orthogonal directions simultaneously for one hour. For the purposes of this work, only the X components that corresponds to the more flexible axes of the buildings were used. To perform the remote measurements, the RAR device was placed at about $50 \mathrm{~m}$ with an inclination of $44^{\circ}$ (building A) and $35^{\circ}$ (building B), with respect to the horizon. RAR antennas were pointing towards the upper vertex of the facades of the buildings. The remote measurements were performed at sampling frequencies of $87.7 \mathrm{~Hz}(\mathrm{~A})$ and $86.2 \mathrm{~Hz}$ (B) for 30 minutes in both cases. In order to obtain good quality signals, avoiding noise, measurements were carefully performed eluding obstacles or objects that could interfere the LOS of the RAR device. Areas where there were no cables, traffic, people or any moving object were preferred. Nevertheless, this was not possible in the case of the B building in which a Tramway line passes through the street between the building and the RAR device. (See Figure 2).

\section{TIME-HISTORY ANALYSES}

Accelerometers and RAR devices can sample at very high frequencies, however, this is only useful if the high frequency content of the signal is of interest. In the case of usual buildings, frequencies of interest are usually in the range from 0.1 to $10 \mathrm{~Hz}$ (periods from 0.1 to $10 \mathrm{sec}-$ onds). Therefore, the as-recorded signals were first resampled to a sampling frequency of 50 $\mathrm{Hz}$, in the case of accelerometers. This way, the data to be analyzed is reduced without affecting the range of frequencies of interest, which is $25 \mathrm{~Hz}$, according to the Nyquist criterion. For RAR signals, time series were homogeneously resampled also to $50 \mathrm{~Hz}$. It is worth noting that the sampling frequency in RAR signals was slightly variable throughout the signal; the mean sampling frequencies were 87.7 and $86.2 \mathrm{~Hz}$ respectively for the A and B buildings. Afterwards, data were filtered with a three-order causal band-pass Butterworth filter to focus the analyses in the frequency band $0.5-10 \mathrm{~Hz}$ (periods in the range $0.1-2 \mathrm{~s}$ ). 
Rodrigo E. Alva, José R. González-Drigo, Guido Luzi, Oriol Caselles, Luís G. Pujades, Yeudy F. Vargas-Alzate, Luis A. Pinzón

\subsection{Accelerometer time-histories records}

Figure 3 shows the one-hour filtered acceleration recordings for the A and B buildings. It can be seen that the amplitudes are greater for the A building because this is taller and more flexible. The magnitude of acceleration is slightly greater in the X directions, which correspond to the flexible axes of both structures. These $\mathrm{X}$ directions were the ones monitored with the RAR system.
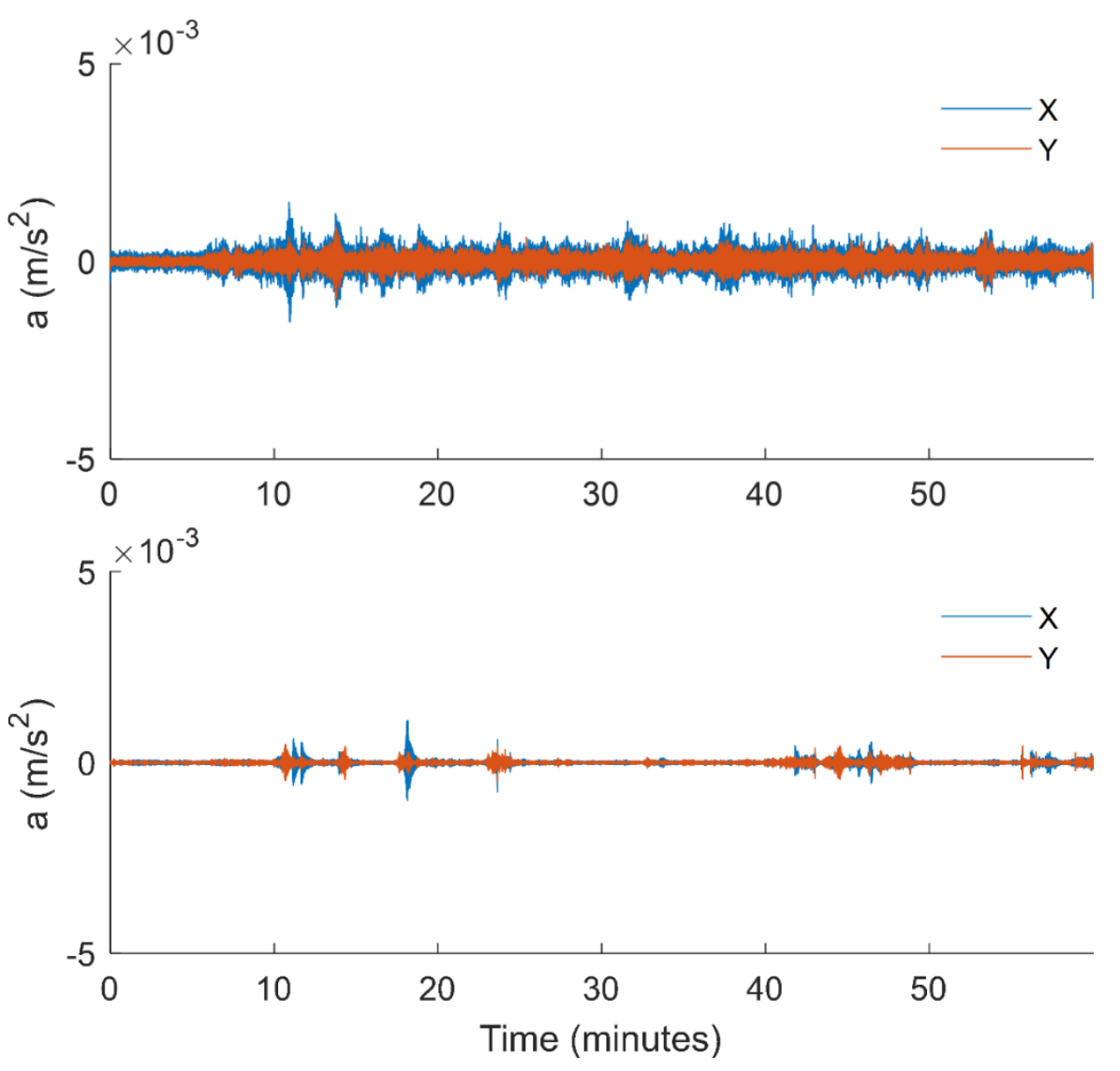

Figure 3: Acceleration records for the A (up) and B (down) buildings. Data have been filtered in the 0.5-10 $\mathrm{Hz}$ band.

In order to find the fundamental frequencies of the structures, the frequency content of the acceleration was analyzed at this phase. To do this, the Power Spectral Density (PSD) method by Welch [7] was calculated. Figure 4 compares the PSD of the A building for both the X (black) and Y (red) directions calculated with a Hamming window of 2621 points (52.42 s) and overlapping of $50 \%$. It can be seen that the peaks of high power are well defined with a resolution of about $0.02 \mathrm{~Hz}$. For the A building (Figure $4-$ up-), the main following frequencies were detected in the $\mathrm{X}$ direction: $0.83,1.13,1.32,2.20$ and 2.49 , which correspond to periods of $1.20,0.88,0.75,0.45$ and $0.40 \mathrm{~s}$, respectively.

In the case of the B building, the PSD was calculated in the same way. Figure 4 (down) shows clear power peaks at frequencies of 1.55 and $1.95 \mathrm{~Hz}$ (Periods of 0.64 , and $0.51 \mathrm{~s}$ ) for the $\mathrm{X}$ direction. 
Rodrigo E. Alva, José R. González-Drigo, Guido Luzi, Oriol Caselles, Luís G. Pujades, Yeudy F. Vargas-Alzate, Luis A. Pinzón

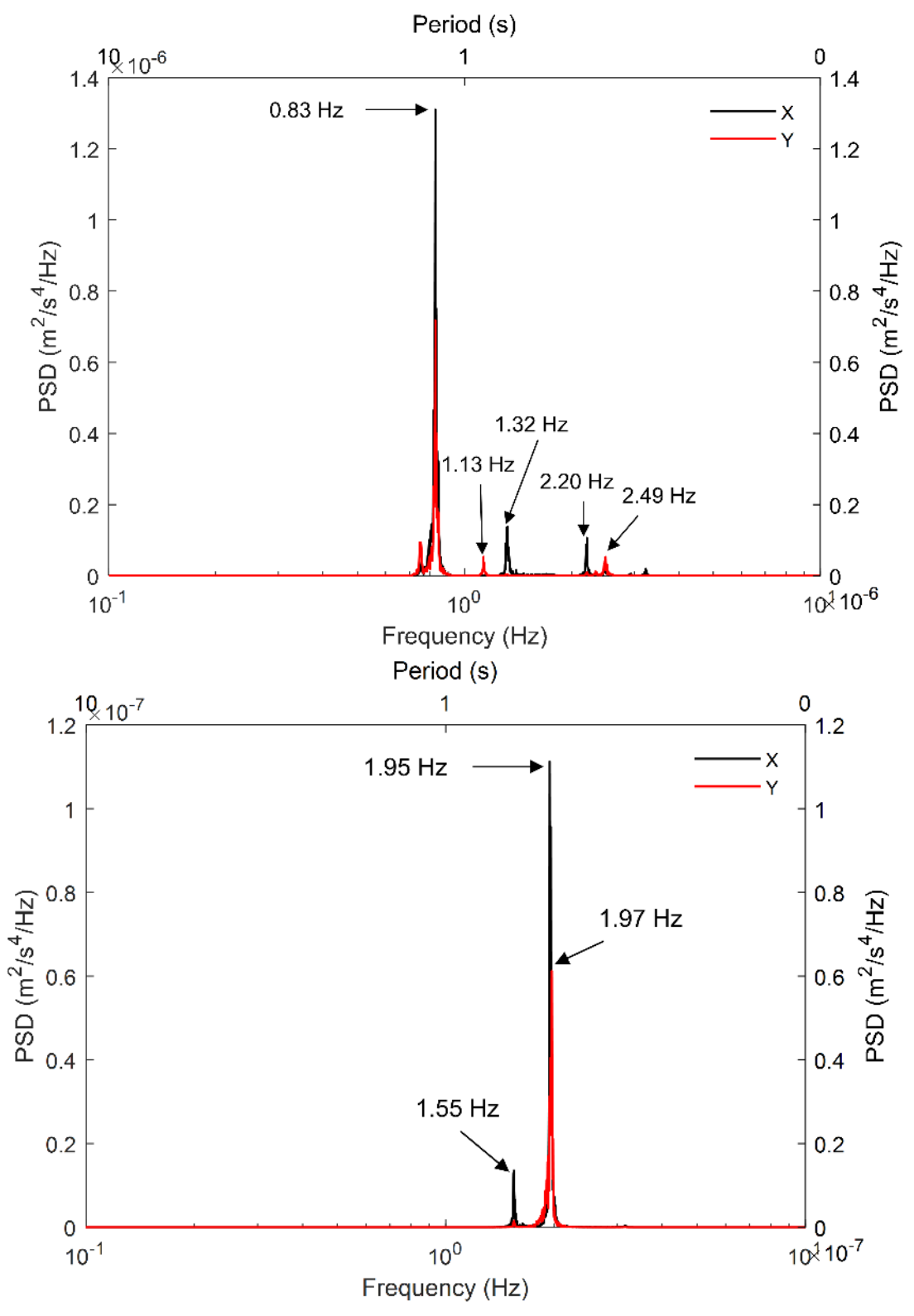

Figure 4: PSD of the acceleration records for the A building, X (black) and Y (red) directions.

To focus on the motion of the building at specific frequencies clearly seen in the PSD graphs, a very narrow band-pass filter is used to isolate the accelerations at these frequencies, both in $\mathrm{X}$ and $\mathrm{Y}$ directions, so that the particle motion at the site of the instrument, can be reproduced. A frequency band of $0.01 \mathrm{~Hz}$ centered at the frequency of interest has been used in these cases. Figure 5 shows the particle motion, for one-hour recordings, in buildings. Frequencies of 0.83 (Figure 5a) and $1.13 \mathrm{~Hz}$ (Figure 5b) are shown for the building A. At $0.83 \mathrm{~Hz}$, building is vibrating in a direction of about $56^{\circ}$ to $\mathrm{Y}$-axis. At $1.13 \mathrm{~Hz}$, the motion is smaller, according to the corresponding PSD (see Figure 4), and mainly in the Y direction. Frequencies of $1.55 \mathrm{~Hz}$ (c) and $1.95 \mathrm{~Hz}$ (d) have been isolated for the B building. Note that at $1.55 \mathrm{~Hz}$ the magnitude of the motion is smaller than the magnitude at $1.95 \mathrm{~Hz}$ and its direction is of about $18^{\circ}$ with the positive direction of the $\mathrm{X}$-axis. The direction of the movement at $1.95 \mathrm{~Hz}$ is of about $-13^{\circ}$ with the positive direction of the X-axis. These motions are related to the modes of vibration of the buildings and indicate that RAR measurement will be more efficient in the X directions. 
Rodrigo E. Alva, José R. González-Drigo, Guido Luzi, Oriol Caselles, Luís G. Pujades, Yeudy F. Vargas-Alzate, Luis A. Pinzón
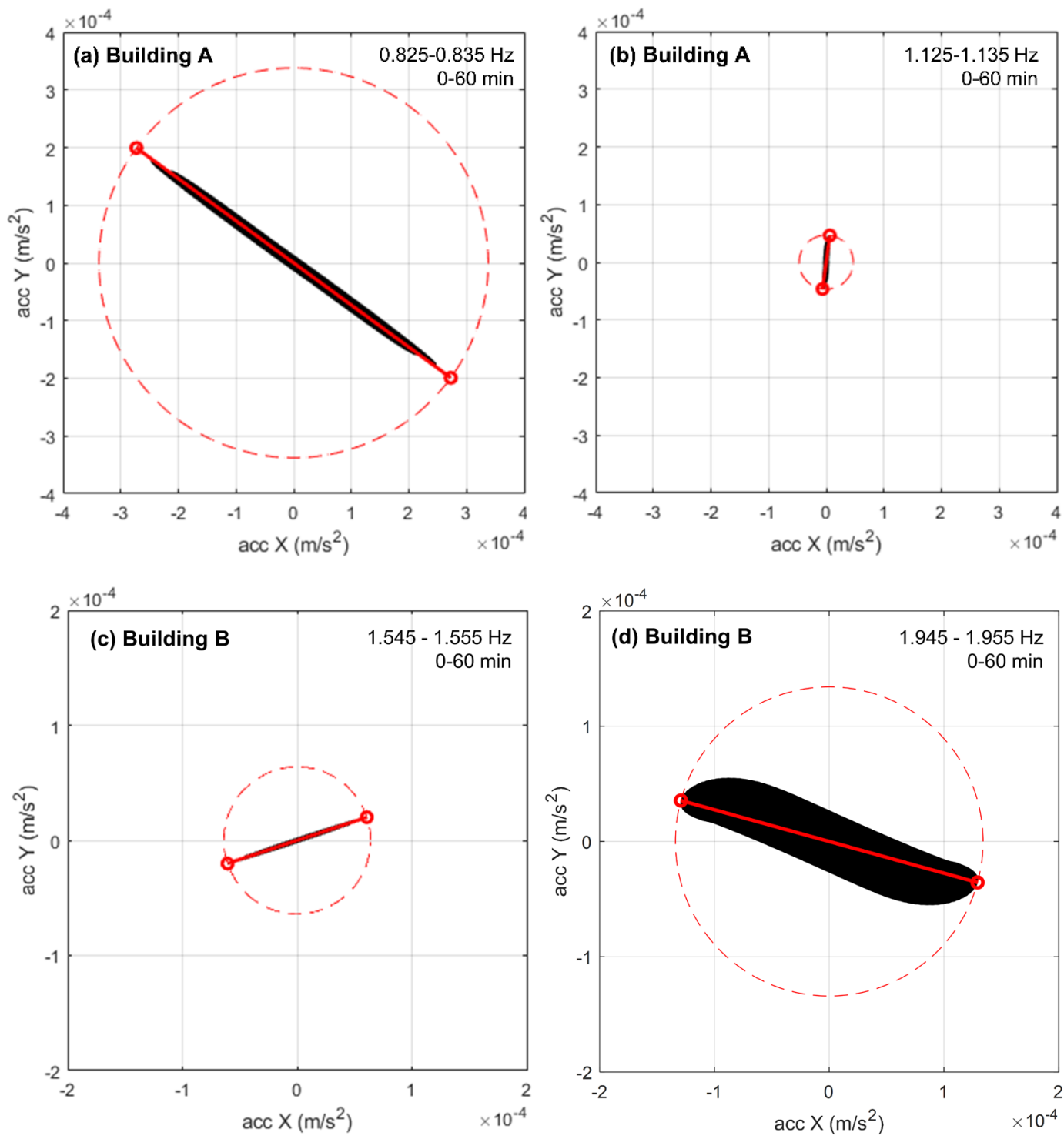

Figure 5: Particle motion, for one-hour recordings at frequencies 0.83 (a) and $1.13 \mathrm{~Hz}$ (b) for building A; and 1.55 (c) and 1.95 (d) for building B. The red continuous line indicates the amplitude and direction of the motion.

\subsection{Displacement time-histories records (RAR)}

The RAR device used performs the simultaneous monitoring of a number of different time series, which correspond to different reflections in the façade of the building at different heights. These different time-series are called Range Bins (Rbins) [8]. Figure 6 shows Rbins for A (up) and B (down) buildings. The RAR system also provides a profile with the Signal-to-Noise Ratio (SNR) for each Rbin (Figure 7), allowing selecting the ones with the highest SNRs corresponding to the range of the buildings. For the A building the Rbins 128 and 138 were selected. For the B building, the Rbins 112 and 120 were chosen. 
Rodrigo E. Alva, José R. González-Drigo, Guido Luzi, Oriol Caselles, Luís G. Pujades, Yeudy F. Vargas-Alzate, Luis A. Pinzón

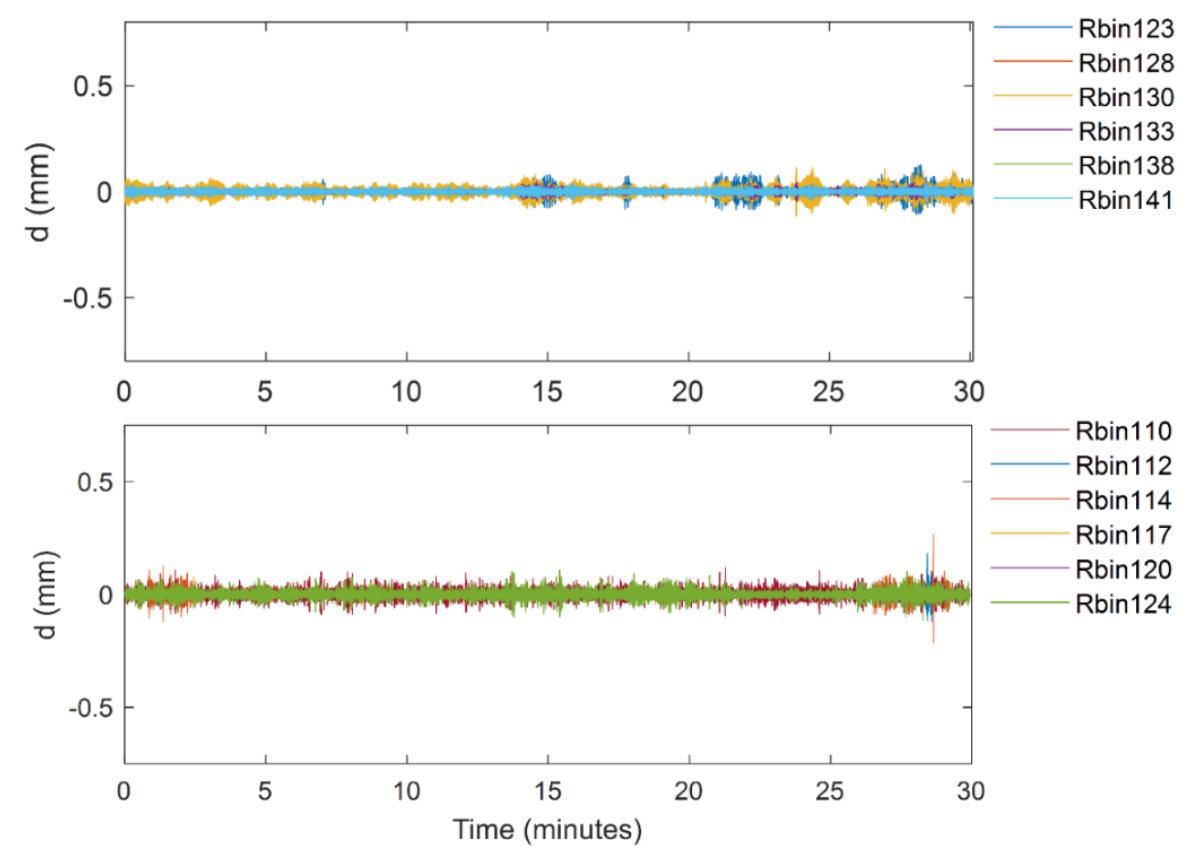

Figure 6: RAR displacement time series for different Rbins recorded in the A (up) and B (down) buildings.
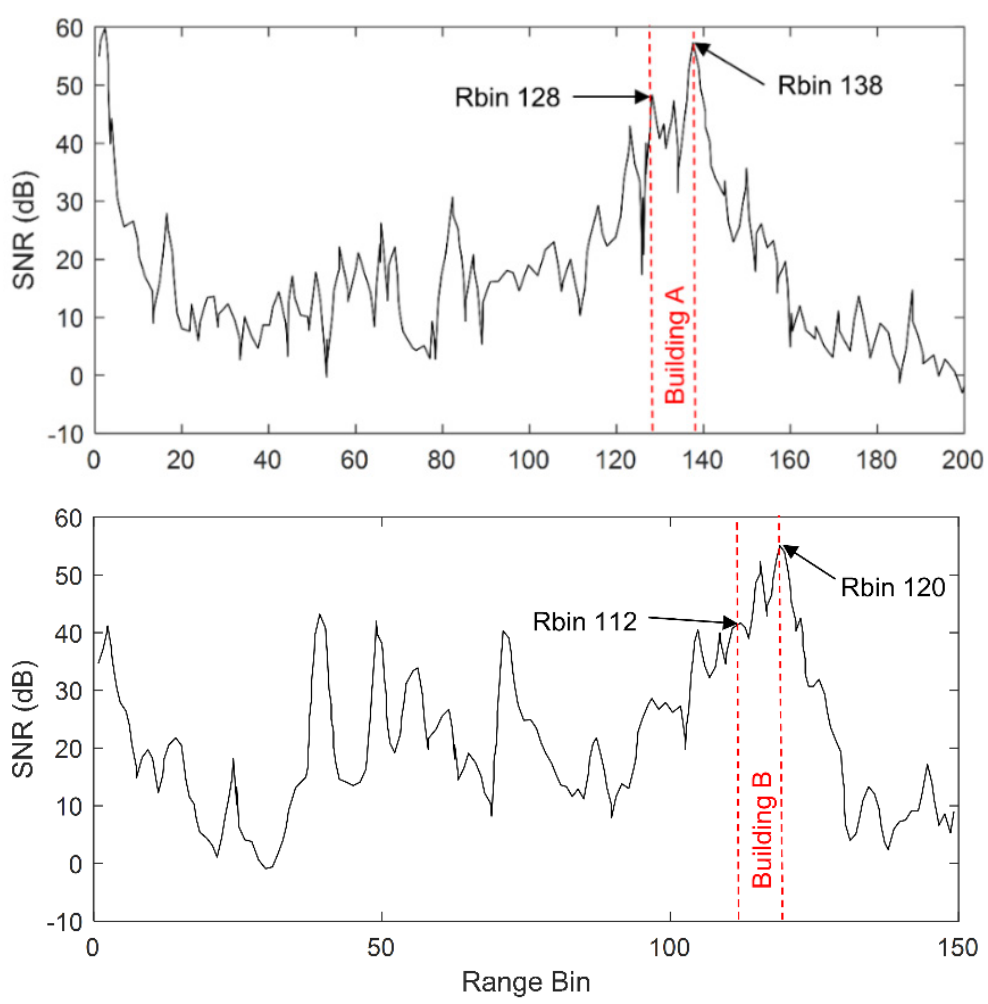

Figure 7: RAR SNR-Range profiles for the A (up) and B (down) buildings.

Figure 8 shows the PSDs for the A building. Frequencies of $0.82,1.13$ and $1.29 \mathrm{~Hz}$, corresponding to periods of $1.21,0.88$ and 0.77 , respectively, were detected. Figure 9 corresponds to the Rbin 128. In this figure, these three peaks can be clearly seen, being the first peak the one with greatest PSD. 
Rodrigo E. Alva, José R. González-Drigo, Guido Luzi, Oriol Caselles, Luís G. Pujades, Yeudy F. Vargas-Alzate, Luis A. Pinzón

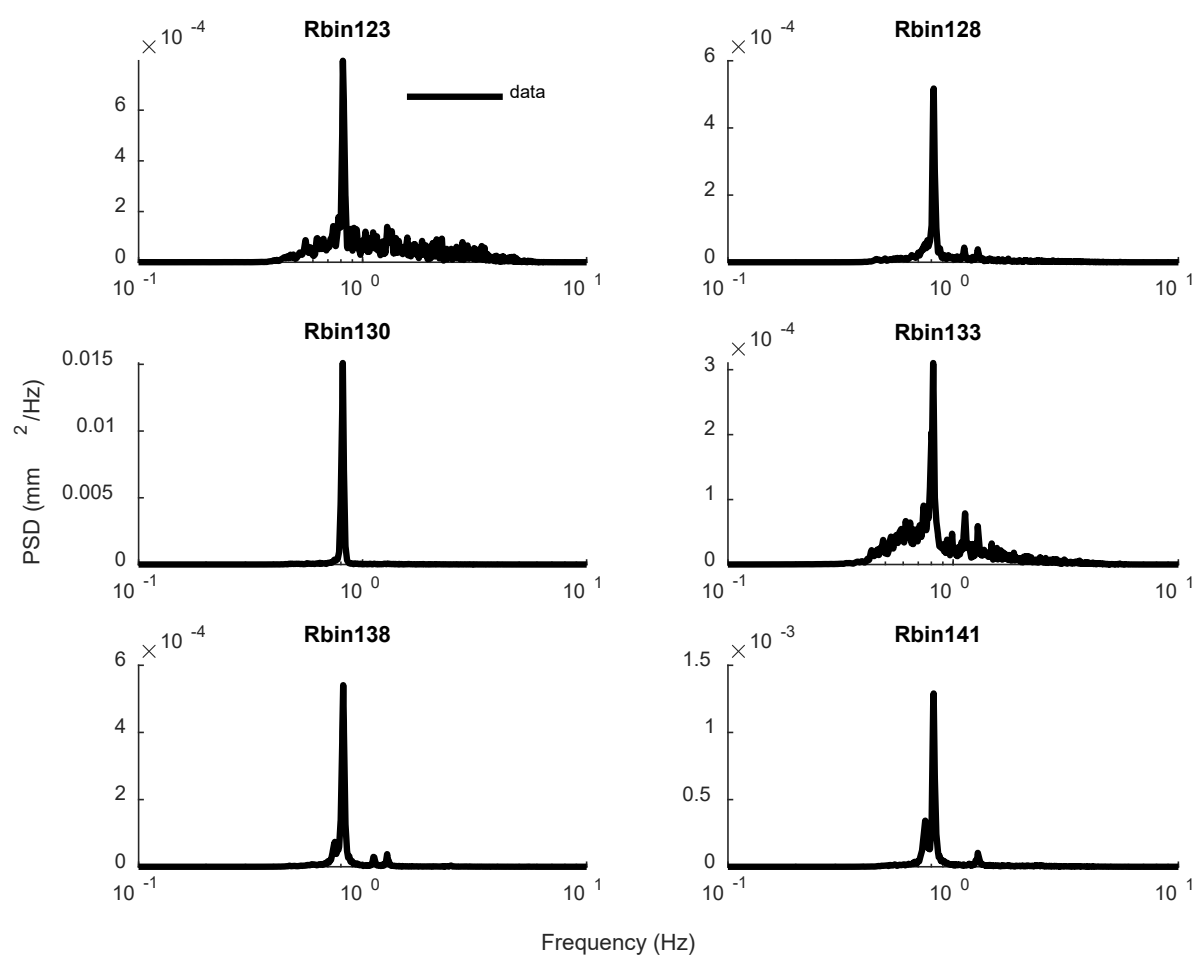

Figure 8: Comparison of the PSDs of the Rbins with good SNRs (A building)

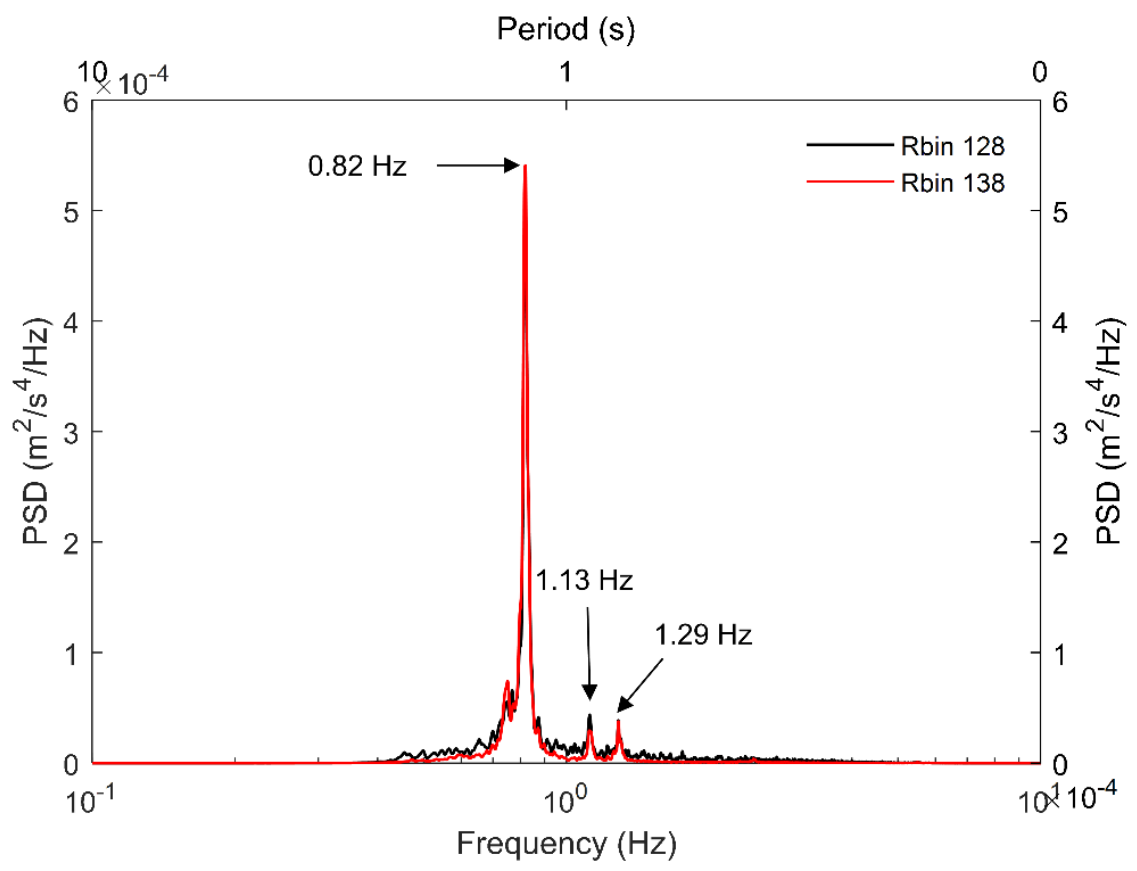

Figure 9: PSD of the Rbins 128 and 138 (A building)

Figure 10 shows the PSD for Rbins corresponding to the B building. Figure 11 shows the PSD of the 112 Rbin, which is the one with the highest SNR. In spite of the noise, a clear PSD peak can be observed at a frequency of $1.98 \mathrm{~Hz}$ (period of about $0.50 \mathrm{~s}$ ). This peak can be also clearly observed at Rbins 117 and 120. 
Rodrigo E. Alva, José R. González-Drigo, Guido Luzi, Oriol Caselles, Luís G. Pujades, Yeudy F. Vargas-Alzate, Luis A. Pinzón
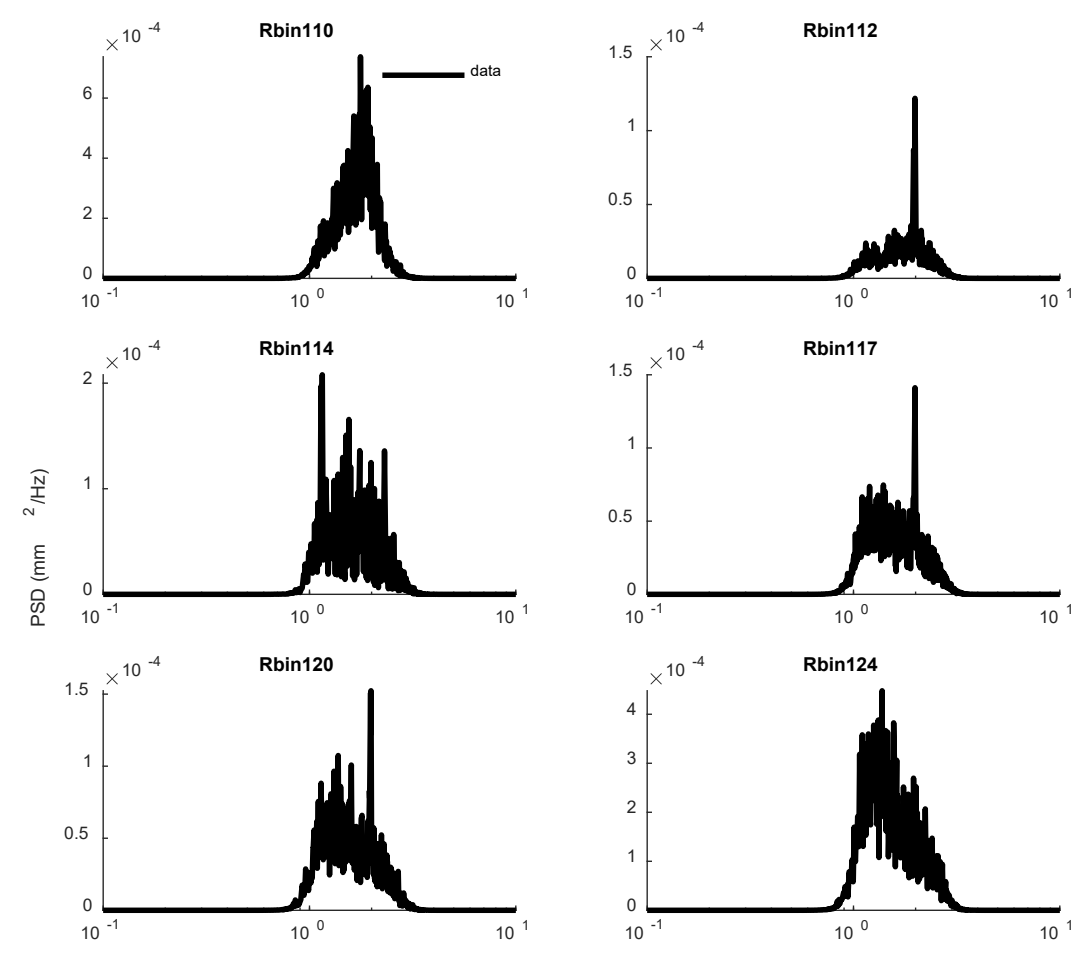

Frequency $(\mathrm{Hz})$

Figure 10: PSD comparison for the better SNR bins of the case B building

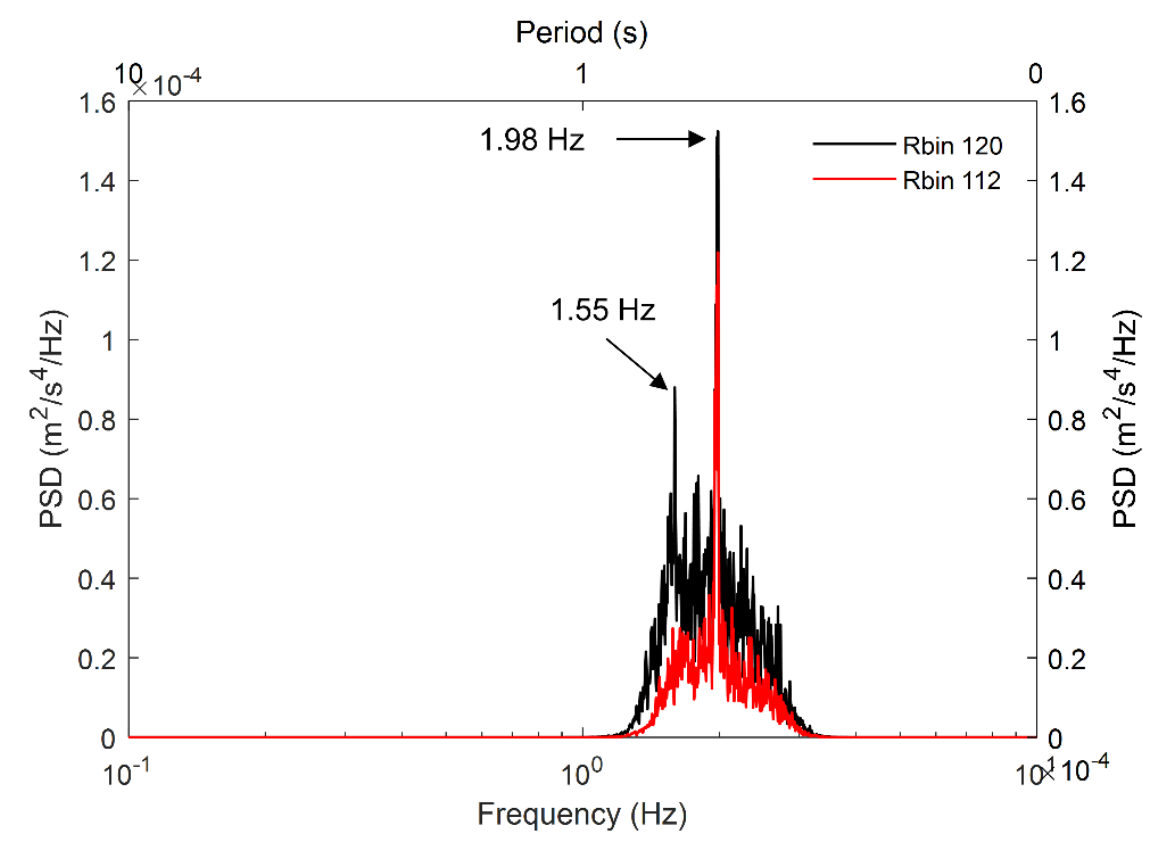

Figure 11: PSD of the Rbins 112 and 120 of the case B building

\section{RAR AND ACCELEROMETER RESULTS COMPARISON}

Six outstanding frequencies have been identified in the A building and two in the B building by means of accelerometric measurements. RAR-based measurements allowed identifying the 
Rodrigo E. Alva, José R. González-Drigo, Guido Luzi, Oriol Caselles, Luís G. Pujades, Yeudy F. Vargas-Alzate, Luis A. Pinzón

main frequencies corresponding to the accelerometer data post-process; three in the A building and two in the B building. Table 1 summarizes the frequencies and periods obtained.

\begin{tabular}{ccccc}
\hline Building & \multicolumn{2}{c}{ Accelerometer } & \multicolumn{2}{c}{ Real-Aperture Radar } \\
frequencies & Periods & $\begin{array}{c}\text { frequencies } \\
(\mathrm{Hz})\end{array}$ & $\begin{array}{c}\text { Periods } \\
(\mathrm{s})\end{array}$ & $\begin{array}{c}\mathrm{Hz}) \\
(\mathrm{s})\end{array}$ \\
\hline \multirow{3}{*}{$\mathrm{A}$} & 0.83 & 1.205 & 0.82 & 1.220 \\
& 1.13 & 0.885 & 1.13 & 0.885 \\
& 1.32 & 0.758 & 1.29 & 0.775 \\
& 2.20 & 0.455 & - & - \\
\hline \multirow{3}{*}{$\mathrm{B}$} & 2.49 & 0.403 & - & - \\
\hline & 1.54 & 0.649 & 1.55 & 0.645 \\
& 1.95 & 0.513 & - & - \\
& 1.96 & 0.510 & 1.98 & 0.505 \\
\hline
\end{tabular}

Table 1: Comparison of frequencies detected with both methods for the A and B buildings.

\section{CONCLUSIONS}

The capability of a RAR-based system for identifying the modal properties of actual buildings has been investigated. Two buildings, one with steel structure and one with RC structure, have been surveyed by considering two approaches: i) accelerometers at the roof of the buildings and ii) a RAR device pointing the façade in the more flexible direction of the surveyed buildings. Noticeably, RAR-based method provided good results for both cases of study. Nonetheless, when RAR is used in high-rise and ductile/flexible structures it is expected an improvement on the quality of the measurements. It should be analyzed how will be the performance of the RAR-based technique when applied to more rigid structures.

Additional work on modelling the surveyed building will provide valuable information about the meaning of the frequencies identified herein which, very likely, will correspond to the fundamental and higher modes of vibration of the building. Of course, seen in another light, the RAR results can be used to diminish the uncertainty related to the structural modelling, i.e. the magnitude of some properties as, for instance, the elastic modulus of the materials, could be better calibrated based on the identification of the modal properties of a structure. Remarkably, the most important conclusion of the present study is that measurements with RAR-based methods are reliable and may be done without the need of entering the buildings; this is an important advantage when the state of safety of a severely damaged structure should be identified.

\section{ACKNOWLEDGMENTS}

This research has been partially funded by the Ministry of Economy and Competitiveness (MINECO) of the Spanish Government and by the European Regional Development Fund (ERDF) of the European Union (EU) through projects with references CGL2015-65913 -P (MINECO/ERDF, EU) and EFA158/16/POCRISC (INTERREG/POCTEFA. EU). The first author, is the holder of a PhD fellowship from the Centro Nacional de Ciencia y Tecnología (CONACyT) in México. Yeudy F. Vargas-Alzate has been granted an Individual Fellowship (IF) in the research grants program of the Marie Sklodowska-Curie Actions (MSCA) of the European Union/European (H2020-MSCA-IF-2017) No 799553. 
Rodrigo E. Alva, José R. González-Drigo, Guido Luzi, Oriol Caselles, Luís G. Pujades, Yeudy F. Vargas-Alzate, Luis A. Pinzón

\section{REFERENCES}

[1] H. Sohn, C.R. Farrar, F.M. Hemez, D.D. Shunk, D.W. Stinemates, B.R. Nadler, J.J. Czarnecki, A review of structural health monitoring literature: 1996-2001. Los Alamos National Laboratory, USA, 2003.

[2] R.M. Goldstein, H.A. Zebker, C.L. Werner, Satellite radar interferometry: Two-dimensional phase unwrapping. Radio science, 23(4), 713-720, 1988.

[3] A. B. Stanbridge, D.J. Ewins, Modal testing using a scanning laser Doppler vibrometer. Mechanical systems and signal processing, 13(2), 255-270, 1999.

[4] D. Tarchi, H. Rudolf, M. Pieraccini, C. Atzeni, Remote monitoring of buildings using a ground-based SAR: application to cultural heritage survey. International Journal of Remote Sensing, 21(18), 3545-3551, 2000 W.R. Alpers, D.B. Ross, C.L. Rufenach, On the detectability of ocean surface waves by real and synthetic aperture radar. Journal of Geophysical Research: Oceans, 86.C7: 6481-6498, 1981.

[5] M. Pieraccini, Monitoring of civil infrastructures by interferometric radar: A review. The Scientific World Journal, 2013.

[6] M. Pieraccini, N. Casagli, G. Luzi, D. Tarchi, D. Mecatti, L. Noferini, C. Atzeni, Landslide monitoring by ground-based radar interferometry: a field-test in Valdarno (Italy). International Journal of Remote Sensing, 24(6), 1385-1391, 2003.

[7] P. Welch, The use of fast Fourier transform for the estimation of power spectra: a method based on time averaging over short, modified periodograms. IEEE Transactions on audio and electroacoustics, 15(2), 70-73, 1967.

[8] C. Negulescu, G. Luzi, M. Crosetto, D. Raucoules, A. Roullé, D. Monfort, T. Dewez, Comparison of seismometer and radar measurements for the modal identification of civil engineering structures. Engineering Structures, 51, 10-22, 2013. 